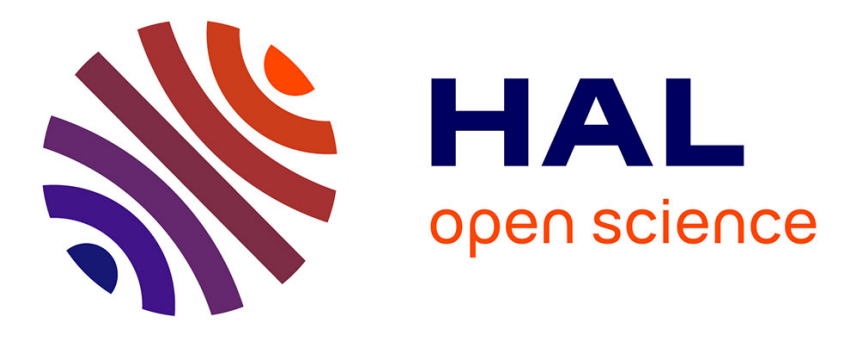

\title{
Spatial distribution of the intensity noise of a vertical-cavity surface-emitting semiconductor laser
}

Alberto Bramati, Jean-Pierre Hermier, A. Z. Khoury, Elisabeth Giacobino, P. Schnitzer, R. Michalzik, K.J. Ebeling, Jean-Philippe Poizat, Philippe Grangier

\section{- To cite this version:}

Alberto Bramati, Jean-Pierre Hermier, A. Z. Khoury, Elisabeth Giacobino, P. Schnitzer, et al.. Spatial distribution of the intensity noise of a vertical-cavity surface-emitting semiconductor laser. Optics Letters, 1999, 24 (13), pp.893-895. 10.1364/OL.24.000893 . hal-00558788

\section{HAL Id: hal-00558788 \\ https://hal-iogs.archives-ouvertes.fr/hal-00558788}

Submitted on 3 Apr 2012

HAL is a multi-disciplinary open access archive for the deposit and dissemination of scientific research documents, whether they are published or not. The documents may come from teaching and research institutions in France or abroad, or from public or private research centers.
L'archive ouverte pluridisciplinaire HAL, est destinée au dépôt et à la diffusion de documents scientifiques de niveau recherche, publiés ou non, émanant des établissements d'enseignement et de recherche français ou étrangers, des laboratoires publics ou privés. 


\title{
Spatial distribution of the intensity noise of a vertical-cavity surface-emitting semiconductor laser
}

\author{
A. Bramati, J.-P. Hermier, A. Z. Khoury, and E. Giacobino \\ Laboratoire Kastler Brossel, Université Pierre et Marie Curie, Ecole Normale Supérieure, Centre National de la Recherche Scientifique, \\ 4 place Jussieu, F-75252 Paris Cedex 05, France \\ P. Schnitzer, R. Michalzik, and K. J. Ebeling \\ Department of Optoelectronics, University of Ulm, Albert-Einstein-Allee 45, D-89069 Ulm, Germany
}

J.-Ph. Poizat and Ph. Grangier

Laboratoire Charles Fabry de l'Institut d'Optique, B.P. 147, F91403 Orsay Cedex, France

Received April 5, 1999

\begin{abstract}
We studied anticorrelated quantum fluctuations between the $\mathrm{TEM}_{00}$ and the $\mathrm{TEM}_{01}$ transverse modes of a vertical-cavity surface-emitting semiconductor laser by measuring the transverse spatial distribution of the laser beam intensity noise. Our experimental results are found to be in good agreement with the predictions of a phenomenological model that accounts for quantum correlations between transverse modes in a light beam. (c) 1999 Optical Society of America

OCIS codes: $250.7260,140.5960,270.6570,230.5440$.
\end{abstract}

During the past few years a major effort has been put into the development of vertical-cavity surfaceemitting semiconductor lasers (VCSEL's). This new type of semiconductor laser has some distinct advantages compared with conventional edge-emitting semiconductor lasers. VCSEL's have a lower threshold, a high quantum efficiency, and a single longitudinal mode. Single-transverse-mode operation reasonably high above threshold has also been observed. ${ }^{1}$

These features make VCSEL's good candidates for use in the generation of amplitude-squeezed light., ${ }^{2,3}$ In general, single-mode operation is more desirable. However, as the driving current is increased, highorder transverse modes may appear. ${ }^{4,5}$ Even in this case, strong anticorrelations between the transverse modes have permitted the realization of amplitudesqueezed light. ${ }^{6}$ Multimode squeezed light has also been observed with longitudinal modes ${ }^{7,8}$ and with polarization modes ${ }^{9}$ in edge-emitting lasers. In semiconductor lasers the homogeneously broadened gain profile permits strong anticorrelations between modes and makes possible radiative emission into different modes without introducing noise into the total laser output photon stream. It must be mentioned, however, that in some cases small inhomogeneities can degrade the anticorrelations and increase the total intensity noise. ${ }^{8}$ Anticorrelations thus play a crucial role in the overall quantum noise of the output laser beam.

We have measured the anticorrelations between transverse modes in a VCSEL by investigating its spatial intensity noise profile. We compared our experimental results with the predictions of a phenomenological quantum model. Only a few measurements of the spatial structure of the intensity noise were already performed, ${ }^{10-14}$ and our investigations enabled us to know the details of this structure with reference to the shot-noise level. We show here that a decrease of noise, and even squeezing, can be obtained when the beam is partially screened.

In our setup the laser beam is screened by a movable razor blade, and the intensity noise is measured as a function of the position $y$ of the blade ${ }^{11}$ (see Fig. 1). In our situation the only two relevant modes are the orthogonally linearly polarized $\mathrm{TEM}_{00}$ and $\mathrm{TEM}_{01}$ modes. As they exhibit different transverse intensity distributions, their respective contributions to the total intensity noise depend on the position of the blade.

To model the processes involved in our experiment we consider a beam composed of two transverse modes, $\mathrm{TEM}_{00}$ and $\mathrm{TEM}_{01}$, with different frequencies and or thogonal linear polarizations. We therefore consider these two modes to be incoherent. To calculate the intensity noise of the detected part of the beam we can model the razor blade as a beam splitter with position-dependent transmissivity $t$ and reflectivity $r$. Because the transverse intensity distribution is

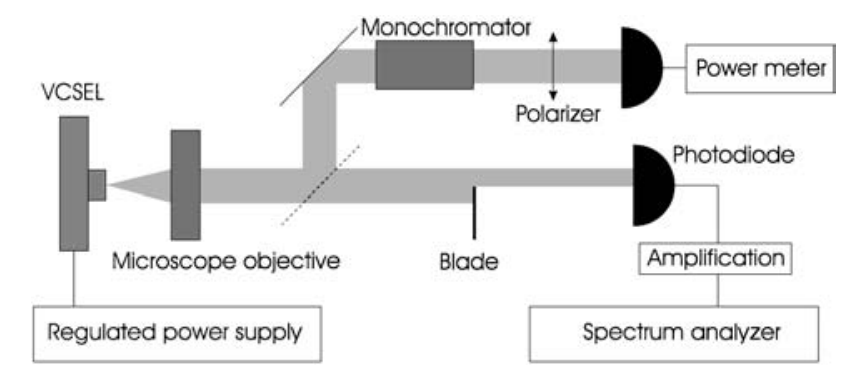

Fig. 1. Experimental setup. The razor blade is mounted upon a motorized translation stage. 
different for the two modes, we must consider different transmissivities and reflectivities for each mode. We denote the photon number operators that correspond to the overall beam and to the $\mathrm{TEM}_{00}$ and $\mathrm{TEM}_{01}$ modes $n, n_{00}$, and $n_{01}$, respectively. The intensity transmissivities for mode $i$ at a given position $y$ of the blade are given by

$$
\begin{aligned}
& T_{00}(y)=\sqrt{2} /(w \sqrt{\pi}) \int_{y}^{+\infty} \exp \left(-2 u^{2} / w^{2}\right) \mathrm{d} u, \\
& T_{01}(y)=4 \sqrt{2} /\left(w^{3} \sqrt{\pi}\right) \int_{y}^{+\infty} u^{2} \exp \left(-2 u^{2} / w^{2}\right) \mathrm{d} u,
\end{aligned}
$$

where $w$ is the size of the beam. The degree of correlation $C$ between the two modes is defined as

$$
C=\frac{\left\langle\delta n^{2}\right\rangle-\left(\left\langle\delta n_{00}^{2}\right\rangle+\left\langle\delta n_{01}^{2}\right\rangle\right)}{2\left(\left\langle\delta n_{00}{ }^{2}\right\rangle\left\langle\delta n_{01}{ }^{2}\right\rangle\right)^{1 / 2}}
$$

where $\delta n$ are the variances of the corresponding quantities. In the case of perfect correlations, $C=$ 1 ; in the case of perfect anticorrelations, $C=-1$. We define the normalized variance $v_{0 i}=\left\langle: \delta n_{i}{ }^{2}:\right\rangle /\left\langle n_{i}\right\rangle$, where :: means that normal ordering is used. The value of $v_{0 i}$ represents excess noise if it is positive and squeezing if it is negative. The ratio between the intensities of the two modes $q$ is defined as $q=$ $\left\langle n_{00}\right\rangle /\left\langle n_{01}\right\rangle$. All the parameters used in this model have a physical significance and can be measured independently.

The total intensity noise $S(y)$ (normalized to the shot noise) when the razor blade is at position $y$ can be calculated by standard methods used for beam splitters ${ }^{15}$ :

$$
\begin{aligned}
S(y)= & \frac{T_{00}(y) q}{T_{00}(y) q+T_{01}(y)}\left[1+T_{00}(y) v_{00}\right] \\
& +\frac{T_{01}(y)}{T_{00}(y) q+T_{01}(y)}\left[1+T_{01}(y) v_{01}\right] \\
& +2 \frac{T_{00}(y) T_{01}(y)}{T_{00}(y) q+T_{01}(y)} C\left[q\left(1+v_{00}\right)\left(1+v_{01}\right)\right]^{1 / 2} .
\end{aligned}
$$

Let us now consider the case of perfect anticorrelations, for which $C=-1$. In Fig. 2 we have plotted the shot-noise level and the nor malized intensity noise versus the position of the blade for the parameters given in the figure. The normalization of the intensity noise is made with the shot noise corresponding to the intensity of the beam, which is a function of the position of the blade. The most interesting feature is that amplitude noise squeezing is possible for some positions of the blade. One can therefore obtain amplitude squeez- ing from a beam that has an intensity noise above the shot noise by partially screening the beam, even if the intensity noise of each transverse mode is above the shot-noise level.

The laser used in the experiment is a high-quantumefficiency oxide confined GaAs/AlGaAs VCSEL (made at the Department of Optoelectronics of the University of $\mathrm{Ulm}^{1}$ ) of $7-\mu \mathrm{m}$ diameter. The top (bottom) mirror has a reflectivity of $99.8 \%$ (99\%). The device has an emission wavelength of $\sim 840 \mathrm{~nm}$. It is driven by a low-noise current source and thermally stabilized with active temperature stabilization (drift as small as $0.01{ }^{\circ} \mathrm{C} / \mathrm{h}$ ). The light beam is collimated by an antireflection-coated microscope objective with a large numerical aperture (N.A., 0.6) to avoid optical losses. The thresholds of the various transverse modes are the following: $I_{\text {th } 0}=1.2 \mathrm{~mA}$ for mode $\mathrm{TEM}_{00}, I_{\text {th1 }}=2.4 \mathrm{~mA}$ for mode $\mathrm{TEM}_{01}$, and $I_{\text {th2 }}=2.8 \mathrm{~mA}$ for the next transverse mode. To measure the intensity noise we use only one photodiode (FND100; bandwidth, $10 \mathrm{kHz}-30 \mathrm{MHz}$; quantum efficiency, 90\%). The shot-noise reference is obtained by balanced detection of a diode laser beam that has a value of intensity noise close to that of the shot noise. The shot noise obtained with this method was in agreement within $0.1 \mathrm{~dB}$ with the noise obtained by thermal light generating the same dc current on the photodiode. The photodiode is connected via an amplifier to a spectrum analyzer. In our experiment we could also perform a spectral analysis of the laser beam with a high-resolution monochromator $(0.03 \mathrm{~nm}$ at $840 \mathrm{~nm}$ ). At the output of the monochromator, a Glan polarizer (extinction ratio, $10^{-4}$ ) allows us to measure the polarization of the modes.

The experimental procedure consists first of the independent measurement of each parameter of Eq. (4). The intensity noise and the intensity of the beam are then recorded versus the position of the blade and compared with the prediction of Eq. (4) with no adjustable parameters.

The electrical driving current is adjusted such that the VCSEL operates with only two transverse modes, i.e., $I_{\text {th } 1}<I_{\text {op }}=2.52 \mathrm{~mA}<I_{\text {th } 2 \text {. Using a }}$ monochromator and a polarizer, we can verify that the $\mathrm{TEM}_{00}$ and the $\mathrm{TEM}_{01}$ modes have linear and mutually

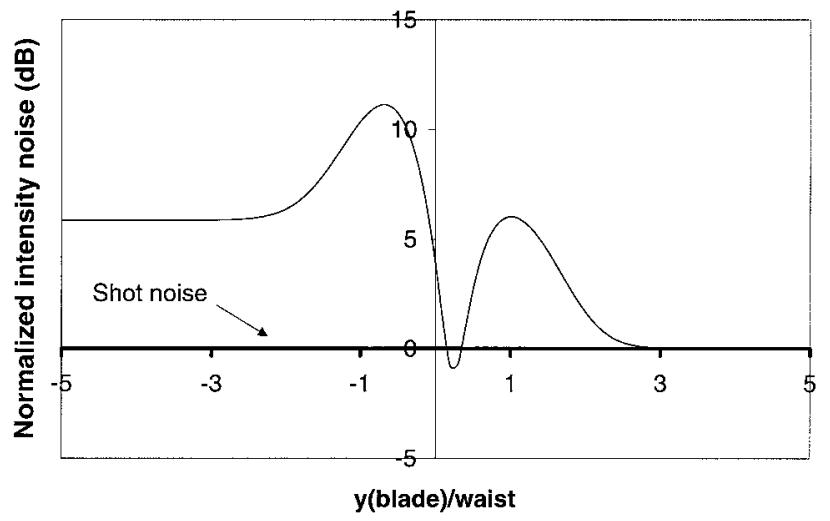

Fig. 2. Normalized intensity noise spectrum versus the position of the razor blade normalized to the size of the beam. $v_{00}=480.6, v_{01}=28.22, C=-1, q=0.11$. 


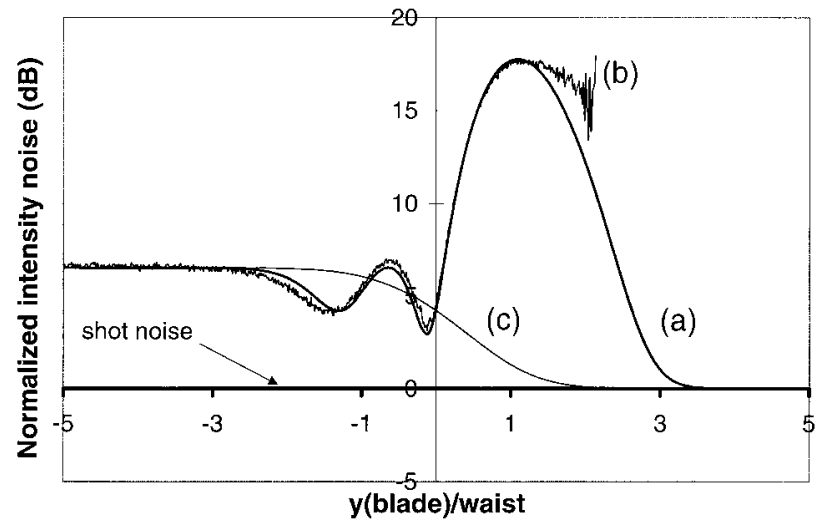

Fig. 3. Normalized intensity noise versus the position of the razor blade normalized to the size of the beam. (a) Theoretical prediction, (b) experimental results. The parameters are $C=-0.98, v_{00}=80.2, v_{01}=3648$, and $q=34.8$. (c) Normalized intensity noise that would be obtained by attenuation of the full beam to the intensity of the cut beam when the blade is at position $y$.

orthogonal polarizations. We measure the intensity of each mode (separating the modes with a Glan polarizer), which gives the values of the quantities $\left\langle n_{00}\right\rangle,\left\langle n_{01}\right\rangle$, and $q$. From the measured intensity noise of each mode we determine the values $v_{00}$ and $v_{01}$ of the excess noise. The measured total intensity noise gives the value of $\left\langle\delta n^{2}\right\rangle$. We then calculate the correlation $C$, using Eq. (3). The size $w$ of the beam is measured when the laser is single mode, i.e., for a driving current $I$ such that $I_{\text {th } 0}<I<I_{\text {th } 1}$.

In Fig. 3 are plotted the experimental results and predictions of our model at an analysis frequency of $15 \mathrm{MHz}$. The agreement between theory and experiment is very good, even though there is a significant uncertainty for the experimental points at the right in Fig. 3. This uncertainty is due to the fact that these points correspond to very low intensities. As expected, because $v_{00}$ and $v_{01}$ are large, strong variations in the intensity noise are observed as the position of the blade is varied. Owing to the correlations between these two modes, it can be seen that the noise is decreased below the normalized noise of the full beam attenuated to the actual intensity of the cut beam [curve (c) of Fig. 3]. This shows that spatial control may be of great importance when it is related to the intensity noise of a VCSEL.

This research was supported by the European Strategic Program for R\&D in Information Technology (ACQUIRE 20029) and the European Community Training and Mobility of Researchers (TMR) "Microlasers and Cavity QED" programs (contract ERBFMRXCT 96-00066). A. Bramati had the support of TMR fellowship ERBFMBI CT950204. J.-P. Hermier's e-mail address is hermier@spectro.jussieu.fr.

\section{References}

1. D. Wiedenmann, P. Schnitzer, C. Jung, M. Grabherr, R. Jager, R. Michalzik, and K. J. Ebeling, Appl. Phys. Lett. 73, 717 (1998).

2. Y. Yamamoto, S. Machida, and O. Nilsson, Phys. Rev. A 34, 4025 (1986).

3. J.-L. Vey and W. Elsässer, Opt. Lett. 23, 721 (1998).

4. J. Martin-Regalado, S. Balle, and M. San Miguel, Opt. Lett. 22, 460 (1997).

5. J. Martin-Regalado, F. Prati, M. San Miguel, and N. B. Abraham, IEEE J. Quantum Electron. 33, 765 (1997).

6. D. C. Kilper, P. A. Roos, and J. L. Carlsten, Phys. Rev. A 55, R3323 (1997).

7. S. Inoue, H. Ohzu, S. Machida, and Y. Yamamoto, Phys. Rev. A 46, 2757 (1992).

8. F. Marin, A. Bramati, E. Giacobino, T.-C. Zhang, J.-Ph. Poizat, J.-F. Roch, and P. Grangier, Phys. Rev. Lett. 75, 4606 (1995).

9. D. C. Kilper, D. G. Steel, R. Craig, and D. R. Scifres, Opt. Lett. 21, 1283 (1996).

10. M. D. Levenson, W. H. Richardson, and S. H. Perlmutter, Opt. Lett. 14, 779 (1989).

11. J.-Ph. Poizat, T. Chang, O. Ripoll, and Ph. Grangier, J. Opt. Soc. Am. B 15, 1757 (1998).

12. G. Giacomelli, F. Marin, M. Gabrysch, K. H. Gulden, and M. Moser, Opt. Commun. 146, 136 (1998).

13. G. Giacomelli and F. Marin, Quantum Semiclassic. Opt. 10, 469 (1998).

14. F. Marin and G. Giacomelli, Quantum Semiclassic. Opt. 1, 128 (1999).

15. M. O. Scully and M. S. Zubairy, Quantum Optics (Cambridge U. Press, Cambridge, 1997), p. 125. 